\title{
Application of nanofilms for arsenic speciation using surface-enhanced Raman spectroscopy (SERS)
}

\author{
V. Liamtsau ${ }^{1,2}$ \& Y. Cai ${ }^{1,2}$ \\ ${ }^{1}$ Department of Chemistry and Biochemistry, Florida International University, Miami, FL, USA \\ ${ }^{2}$ Southeast Environmental Research Center, Florida International University, Miami, FL, USA
}

\begin{abstract}
The novel analytical method based on combination of "coffee ring" effect and Surface-enhanced Raman spectroscopy (SERS) has been developed for the speciation of thiolated arsenicals. The gold nanofilm (AuNF) has been fabricated by the coating of glass surface with 3-aminopropyltrimetoxysiloxane (APTMS) and citrate derived negatively charged gold nanoparticles. After the deposition of thioarsenicals buffer solution onto AuNF, following solvent evaporation, ring stamp was formed. Due to the difference in charges of molecules, DMMTA $(\mathrm{V})$ and DMDTA(V) travelled different distances. Overall, the "coffee ring" effect has a decent potential for the speciation of arsenic compounds.
\end{abstract}

\section{INTRODUCTION}

The "coffee ring" effect has been previously employed primarily for the analytes preconcentration, however with proper glass surface modification it could have a potential for the separation of arsenic species. Dimethylmonothioarsinic acid (DMMTA(V)) and dimethyldithioarsinic acid (DMDTA(V)) are thioarsenicals that has been widely detected in animal urine after exposure to iAs(III) and DMA(V) (Fig. 1). Pentavalent thioarsenicals can bind to the proteins in contrast to iAs(III) and DMA(V), thus the toxicities of thioarsenicals are similar with that of iAs(III) and are much higher than those of DMA(V). So, high toxicity and protein binding capacity demonstrate that thioarsenicals might be the key arsenic metabolites related to the As overall toxicity. As a result, it is vital to develop a method that can precisely determine the exact amount of thioarsenicals in biological systems.

\section{METHODS/EXPERIMENTAL}

DMMTA(V) was synthesized according Cullen et al. (2016), the molar ratio of $\mathrm{Na}_{2} \mathrm{~S} / \mathrm{H}_{2} \mathrm{SO}_{4} / \mathrm{DMA}$ was 1.6:1.6:1.

DMDTA(V) was synthesized by two different methods. The first method reported by Suzuki et al. (2004) provided the acidic form of DMDTA $(\mathrm{V})$ in contrast to the second approach that has been described by Fricke et al. (2005) and allowed to synthesize the salt form of DMDTA $(\mathrm{V})$. The molar ratio for these two methods of $\mathrm{Na}_{2} \mathrm{~S} / \mathrm{H}_{2} \mathrm{SO}_{4} / \mathrm{DMA}(\mathrm{V})$ was $7.5: 7.5: 1$.

The preparation of a gold nanofilm includes two stages, synthesis of citrate-coated gold and gold nanoparticles (AuNPs) and coating nanoparticles

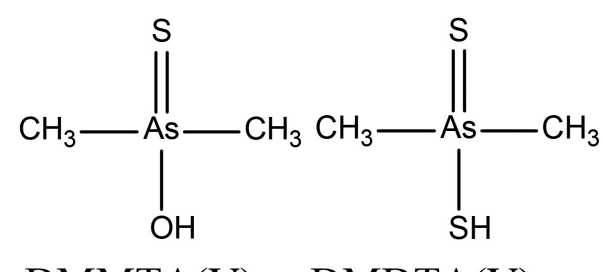

DMMTA(V) DMDTA(V)

Figure 1. The structures of Dimethylmonothioarsinic (DMMTA(V)) acid and dimethyldithioarsinic acid (DMDTA(V))

onto silicon wafers. AuNPs were synthesized by Turkevich method. The fabrication of AuNF was performed by silanization of glass substrates by 3-aminopropyltrimetoxysiloxane (APTMS), following the deposition of negatively charged gold nanoparticles onto the positively charged amino groups silanized surface.

DMMTA(V) and DMDTA(V) solutions $\left(10^{-4} \mathrm{M}\right)$ solutions of thioarsenicals were prepared by dissolving thioarsenicals in buffer, following the deposition of $2 \mu \mathrm{L}$ of DMMTA(V) and DMDTA(V) buffer solution onto the AuNF. Once the droplet was completely dried and a ring-shaped stain was formed on the AuNF, SERS signals were obtained from the center to the edge of the dried droplet.

\section{RESULTS AND DISCUSSION}

Raman spectra of solid thioarsenicals are shown in Figures 2 and Figures 3.

Since charged and neutral species would have various interactions with negatively charged SERS 


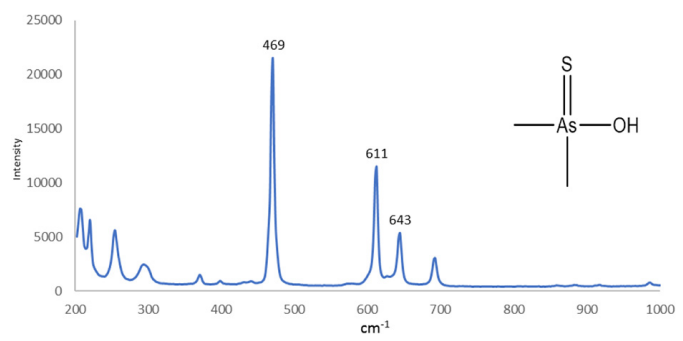

Figure 2. Raman spectroscopy of DMMTA(V).

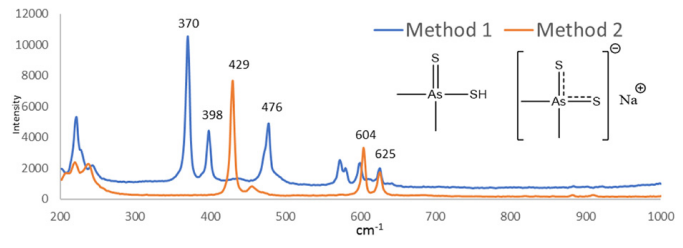

Figure 3. Raman spectroscopy of DMDTA(V).

Table 1. Raman vibrational frequencies of DMMTA(V) and DMDTA(V).

\begin{tabular}{llc}
\hline Arsenical & Assignment & $\begin{array}{l}\text { Experimental Raman } \\
\text { frequency }\left(\mathrm{cm}^{-1}\right)\end{array}$ \\
\hline DMMTA(V) & As $=\mathrm{S}$ & 469 \\
& As $-\mathrm{C}$ & 641 \\
& As $-\mathrm{O}$ & 643 \\
DMDTA(V) & As $-\mathrm{S}$ & 370,398 \\
& As $\div$ S & 429 \\
& s C - As $-\mathrm{C}$ & 573,604 \\
& a C - As $-\mathrm{C}$ & 625 \\
\hline
\end{tabular}

surface, their travelling distances might be different (Table 1). AuNF was applied for the separation of DMMTA(V) and DMDTA(V).

From the graph it is clear that As-S vibrations shift from $429 \mathrm{~cm}^{-1}$ to $417 \mathrm{~cm}^{-1}$ for DMDTA(V) and from $469 \mathrm{~cm}^{-1}$ to $465 \mathrm{~cm}^{-1}$ for DMMTA(V) respectively (Fig. 4), thus, thioarsenicals were adsorbed onto the AuNF and charge transfer between the molecules and AuNF surface occurred. However, it is not clear whether a capillary force that drown thioarsenicals to the edges of evaporating droplet or attraction/repulsion interaction between analytes and the AuNF surface had the major impact onto the separation of thioarsenicals.

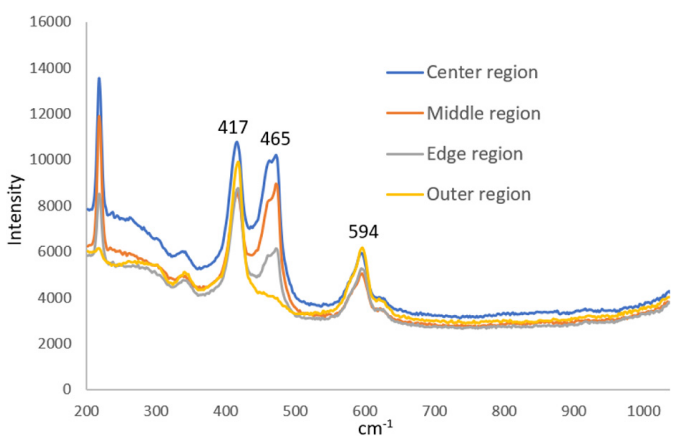

Figure 4. SERS of $10^{-4} \mathrm{M}$ DMMTA(V) and DMDTA(V) buffer solution separated by "coffee ring" effect.

\section{CONCLUSIONS}

DMMTA(V) and DMDTA(V) were separated using "coffee ring" effect and identified by SERS. Nanofilms are versatile tools, facile to fabricate and easy to operate under necessary conditions. Overall, nanofilms had a decent potential for the separation of arsenic species.

\section{ACKNOWLEDGEMENTS}

I would like to thank Dr. Guangliang Liu and Dr. Changjun Fan from the Department of Chemistry and Biochemistry at FIU for their faith in my project.

\section{REFERENCES}

Cullen, W.R., Liu, Q., Liu, X., McKnight-Whitford, A., Peng, H., Popowich, A., Yan, X., Zhang, Q., Fricke, M., Sun., H. \& Le, C. 2016. Methylated and thiolated arsenic species for environmental and health research - a review on synthesis and characterization. J. Environ. Sci. 49: 7-27.

Fricke, M.W., Zeller, M., Sun, H., Lai, V W.-M., Cullen, W.R., Shoemaker, J.A., Witkowski, M.R. \& Creed, J.T. 2005. Chromatographic separation and identification of products from the reaction of dimethylarsinic acid with hydrogen sulfide. Chem. Res. Toxicol. 18(12): 1821-1829.

Suzuki, K.T., Mandal, B.K., Katagiri, A., Sakuma, Y., Kawakami, A., Ogra, Y., Yamaguchi, K., Sei, Y., Yamanaka, K., Anzai, K., Ohmichi, M., Takayama, H. \& Aimi, N. 2004. Dimethylthioarsenicals as arsenic metabolites and their chemical preparations. Chem. Res. Toxicol. 17(7): 914-921. 